\title{
A phase III study of adjuvant chemotherapy in patients with completely resected, node-negative non-small cell lung cancer (JCOG 0707)
}

Hideo Kunitoh, MD, ${ }^{\mathrm{a}}$ Masahiro Tsuboi, MD, ${ }^{\mathrm{b}}$ Masashi Wakabayashi, MSc, ${ }^{\mathrm{c}}$ Morihito Okada, MD, ${ }^{\mathrm{d}}$ Kenji Suzuki, MD, ${ }^{\mathrm{e}}$ Shun-ichi Watanabe, MD, ${ }^{\mathrm{f}}$ and Hisao Asamura, $\mathrm{MD},{ }^{\mathrm{g}}$ on behalf of the Japan Clinical Oncology Group Lung Cancer Surgical Study Group (JCOG-LCSSG)

\section{ABSTRACT}

Objective: To evaluate efficacy of S-1 (tegafur/gimeracil/oteracil), an active novel fluoropyrimidine, as compared to UFT (tegafur/uracil) as a postoperative adjuvant therapy in patients with node-negative non-small cell lung cancer (NSCLC).

Methods: Eligible patients had undergone complete resection of p-stage I ( $T_{1}$ with tumor diameter $>2 \mathrm{~cm}$ or T2-NoMo by 5 th edition Union for International Cancer Control TNM) NSCLC, and were randomized to receive oral UFT $250 \mathrm{mg} / \mathrm{m}^{2} /$ day for 2 years (Arm A) or oral S-1 $80 \mathrm{mg} / \mathrm{m}^{2} /$ day for 2 weeks with a 1-week rest period, for 1 year (Arm B). The primary end point was relapse-free survival (RFS), with $80 \%$ power and a one-sided type I error of 0.05 .

Results: From November 2008 to December 2013, 963 patients were enrolled (Arm A: 482, Arm B: 481). Toxicities (hematologic/nonhematologic) of grade 3 or more were observed in 15.9 (1.5/14.7)\% in Arm A, and in 14.9 (3.6/12.1)\% in Arm $B$, respectively. At data cut-off in December 2018, the hazard ratio for RFS was 1.06 (95\% confidence interval, $0.82-1.36$ ), showing no superiority of S-1 over UFT. The hazard ratio of overall survival (OS) was 1.10 (95\% confidence interval, 0.81-1.50). The 5-year RFS/OS were $79.4 \% / 88.8 \%$ in Arm A and $79.5 \% / 89.7 \%$ in Arm B, respectively. The original NSCLC accounted for $58 \% / 53 \%$, respectively, of the Arm A/Arm B OS events. Secondary malignancies were observed in $85(17.8 \%)$ and $84(17.8 \%)$ individuals in Arm A and Arm B, respectively.

Conclusions: S-1 was not superior to UFT as postoperative adjuvant therapy in node-negative NSCLC. Future investigation should incorporate identification of high-risk populations for recurrence. (JTCVS Open 2020;4:90-102)

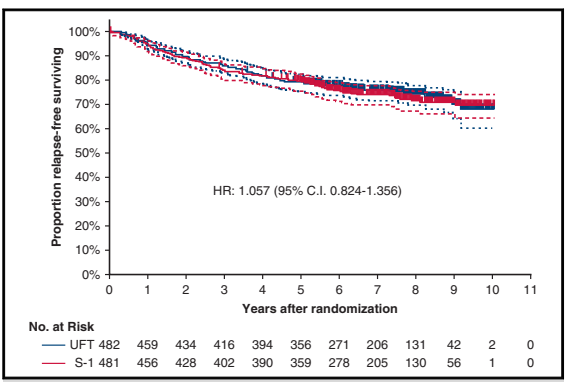

Relapse-free survival, the primary end point of the trial, of all enrolled patients.

\section{CENTRAL MESSAGE}

S-1 (tegafur/gimeracil/oteracil) is an active novel fluoropyrimidine, but S-1 as adjuvant therapy failed to improve the outcome of patients with node-negative nonsmall cell lung cancer after surgery.

\section{PERSPECTIVE}

Outcome of patients with node-negative lung cancer is very good with Japanese standard of care, with 5 -year overall survival of nearly $90 \%$ In addition, there were significant competing risks, such as noncancer deaths and secondary malignancies. Therefore, future research should incorporate identification of, and be focused on, the patient groups at high risk for disease recurrence.

See Commentaries on pages 103 and 105.
From the ${ }^{\mathrm{a} J a p a n e} \mathrm{se}$ Red Cross Medical Center, Tokyo; ${ }^{\mathrm{b}}$ National Cancer Center Hospital East, Kashiwa; 'Japan Clinical Oncology Group Data Center, National Cancer Center Hospital, Tokyo; ${ }^{\mathrm{d}}$ Hiroshima University Hospital, Hiroshima City Minamiku; ' Juntendo University, Tokyo; ${ }^{\mathrm{f}}$ National Cancer Center Hospital, Tokyo; and ${ }^{\mathrm{g}}$ Keio University, Tokyo, Japan.

The study was supported in part by the National Cancer Center Research and Development Funds (20S-2, 20S-6, 23-A-16, 23-A-18, 26-A-4, 29-A-3) from the Ministry of Health, Labour and Welfare of Japan.

Received for publication Aug 16, 2020; revisions received Aug 16, 2020; accepted for publication Aug 18, 2020; available ahead of print Sept 22, 2020.
Address for reprints: Hideo Kunitoh, MD, Department of Medical Oncology, Japanese Red Cross Medical Center, 4-1-22 Hiro-o, Shibuya-ku, Tokyo 150-8935, Japan (E-mail: kunito_hideo@med.jrc.or.jp).

2666-2736

Copyright $(2020$ The Authors. Published by Elsevier Inc. on behalf of The American Association for Thoracic Surgery. This is an open access article under the CC BY-NCND license (http://creativecommons.org/licenses/by-nc-nd/4.0/).

https://doi.org/10.1016/j.xjon.2020.08.009 


$$
\begin{aligned}
& \text { Abbreviations and Acronyms } \\
& \text { CI = confidence interval } \\
& \mathrm{CSPOR}=\text { Comprehensive Support Project for } \\
& \text { Oncology Research } \\
& \mathrm{CT}=\text { computed tomography } \\
& \text { DPD = dihydro-pyrimidine dehydrogenase } \\
& \text { HR = hazard ratio } \\
& \text { JCOG = Japan Clinical Oncology Group } \\
& \text { NO = node-negative } \\
& \mathrm{NSCLC}=\text { non-small cell lung cancer } \\
& \text { OS = overall survival } \\
& \text { PET = positron emission tomography } \\
& p \text {-stage }=\text { pathologic stage } \\
& \text { RFS = relapse-free survival } \\
& \mathrm{S}-1=\text { tegafur/gimeracil/oteracil } \\
& \text { UFT }=\text { tegafur/uracil }
\end{aligned}
$$

Video clip is available online.

Postoperative adjuvant therapy remains controversial for patients with node-negative (NO) non-small cell lung cancer (NSCLC) after surgical resection. Meta-analysis of platinum-based adjuvant therapy suggested its benefit in hilar node-positive and mediastinal node-positive patients with NSCLC. However, no significant benefit was observed in patients with pathologic stage ( $p$-stage) IB (N0 and tumor $>3 \mathrm{~cm}$ ), and there was even a trend toward harm in patients with $p$-stage IA (N0 and tumor $3 \mathrm{~cm}$ or less). ${ }^{1}$ Although suggestions are made for the benefit of platinum-based adjuvant chemotherapy in patients with $\mathrm{N} 0$ and a tumor size $>4 \mathrm{~cm}$, they are based on unplanned subset analyses of randomized trials alone. ${ }^{2,3}$

In Japan, a series of randomized trials suggested a survival benefit of adjuvant therapy with the oral drug tegafur/uracil, or UFT, in patients with NO NSCLC after complete resection. ${ }^{4-7}$ UFT is a fluoropyrimidine drug with inhibition of dihydro-pyrimidine dehydrogenase, or DPD, which is a rate-limiting enzyme in the catabolism of fluorouracil and is associated with drug resistance. Although UFT is only marginally effective in advanced NSCLC, with response rate of only $6 \$$ to $7 \%,{ }^{8,9}$ its efficacy for postoperative adjuvant therapy in NO NSCLC was documented by a large randomized study by Kato and colleagues ${ }^{7}$ and confirmed by a meta-analysis by Hamada and colleagues. ${ }^{10}$ The meta-analysis showed that UFT was beneficial in patients with tumor size $>2 \mathrm{~cm}$. No significant interaction was observed between histology and UFT effect.
Tegafur/gimeracil/oteracil, or S-1, is a novel, more potent DPD-inhibitory fluoropyrimidine. ${ }^{11,12} \mathrm{~S}-1$ is active against a broad spectrum of tumors, including NSCLC, with a single-agent response rate exceeding $20 \%{ }^{13-15}$ Its original administration schedule was 4 weeks on and 2 weeks off, but subsequent studies reported more favorable toxicity files and drug adherence with a modified schedule of 2 weeks on and 1 week off. ${ }^{16}$ Postoperative adjuvant therapy with $\mathrm{S}-1$ for 1 year improved survival outcomes of patients with gastric cancer (as compared with surgery alone $)^{17}$ or with pancreatic cancer (as compared to gemcitabine). ${ }^{18}$ In addition, S-1 was superior to UFT as adjuvant therapy in terms of relapse-free survival in stage II/III rectal cancer. ${ }^{19}$ Due to the mainly gastrointestinal toxicities of S-1, continuation of adjuvant therapy is hardly tolerable beyond 1 year, unlike UFT.

Based on the aforementioned rationales, we, Japan Clinical Oncology Group Lung Cancer Surgical Study Group (JCOG-LCSSG), conducted JCOG 0707 study, a randomized trial of adjuvant S-1 for 1 year versus standard UFT for 2 years, in patients with NO NSCLC with tumor size $>2 \mathrm{~cm}$ after complete surgical resection. The study objectives were to evaluate and safety of S-1 as compared with UFT to test the null hypothesis that there is no difference in relapse-free survival (RFS) between the arms. Since both belong to the same drug class, the clinical question should be, "does a more active (but toxic) drug do better" as adjuvant therapy for early-stage, NO NSCLC. A part of the study results were presented at the World Conference on Lung Cancer on September 8, 2019 (Video 1).

\section{METHODS}

\section{Study Design}

This is an open-label, randomized phase III superiority trial in patients with NO NSCLC after surgical complete resection to confirm the superior

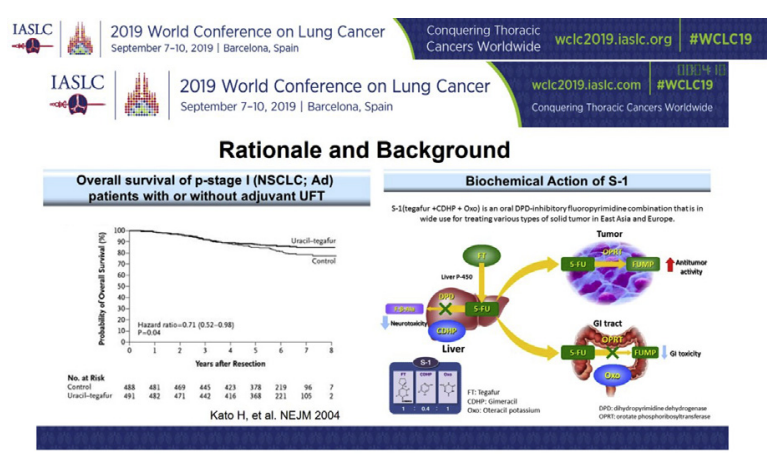

VIDEO 1. The first author, Hideo Kunitoh, presented a part of the study results at the World Conference on Lung Cancer on September 8, 2019, at the mini-oral session "Challenges in the treatment of early stage NSCLC." He emphasized the importance of "cost and value" issue at the conclusion of the presentation. The meeting was held in Barcelona, Spain, during September 7-10, 2019. Video available at: https://www.jtcvs.org/ article/S2666-2736(20)30082-6/fulltext. 
efficacy of adjuvant S-1 therapy over the standard of care, UFT adjuvant therapy. This study was compliant with the ethical principles of the Declaration of Helsinki. The trial protocol was approved by the institutional review boards of all participating institutions (approved by JCOG Committee Office on September 8, 2008). All patients provided informed consent in writing before enrollment. The trial was registered in the UMIN Clinical Trials Registry (UMIN000001494).

\section{Patients}

Patients were eligible for the study if they had histologically documented NO NSCLC (based on the World Health Organization histologic classifications of 1999$)^{20}$ and had undergone complete surgical resection within 56 days of enrollment. Computed tomography (CT) and/or magnetic resonance imaging of the brain or whole-body positron emission tomography (PET) scan was not mandatory at presurgical staging procedures.

Eligibility criteria also included (1) pathologic stage I, as defined by the 5th edition (1997) of Union for International Cancer Control TNM staging system $^{21}$; in cases with $p$-stage IA, the tumor diameter had to be more than $2 \mathrm{~cm}(\mathrm{~T}>2 \mathrm{~cm})$. (2) Tumor resected with lobectomy, bi-lobectomy, or pneumonectomy, with lymph node dissection for ND2a extent (ie, systemic hilar and mediastinal node dissection, excluding anterior and posterior nodes), or elective dissection. (3) No previous therapy except for surgical resection. (4) Eastern Cooperative Oncology Group Performance Status of 0 or 1 with adequate bone marrow, liver, and kidney function. (5) Oxygen saturation of $90 \%$ or more on ambient air. (6) Age 20 to 80 years. (7) Signed informed consent obtained from the patient.

The exclusion criteria and details of the eligibility criteria are provided in Online Data Supplement.

On May 17, 2011, when 551 patients were randomized, patient enrollment was temporarily suspended following a report of the third treatmentrelated death in S-1 arm. The study was resumed on August 1, 2011, with a protocol amendment to tighten exclusion criteria. It was then specified that treatment-related death rate of $1 \%$ or more would be unacceptable in this good-risk patient group. The amended protocol also required strict informed consent, emphasizing the risks associated with S-1 therapy.

\section{Randomization and Masking}

Eligible patients were randomly assigned (1:1) to receive adjuvant S-1 therapy or UFT therapy. The JCOG Data Center performed this randomization using the minimization method to balance for institution, pathologic stage (IA or IB), histology (adenocarcinoma/not adenocarcinoma), age (below 65/65 or older), and sex (male or female). Investigators and patients were not masked to treatment allocation.

\section{Treatment}

In Arm A (UFT arm), oral UFT $250 \mathrm{mg} / \mathrm{m}^{2} /$ day (tegafur dose) was administered in 2 or 3 doses per day, for 2 years. It was given continuously, without rest period. In Arm B (S-1 arm), Oral S-1 (Taiho Pharmaceutical Company, Tokyo, Japan) $80 \mathrm{mg} / \mathrm{m}^{2} /$ day (tegafur dose) was administered in 2 doses per day continuously for days 1 to 14 followed by a rest period for days 15 to 21 ( 1 treatment cycle consists of 21 days), for 1 year.

Adverse events of the treatments were evaluated with the Common Terminology Criteria for Adverse Events, version 3.0. ${ }^{22}$ Treatment was suspended with the onset of clinically relevant toxicities and resumed upon on recovery. The dosage was modified according to the criteria in the attached protocol.

In both arms, patients were regularly followed for the complete blood count, including neutrophil count, blood chemistry, oxygen saturation, physical examination, and drug adherence, as defined by the study calendar in the attached protocol. A chest radiograph was taken monthly for the first 3 months, tri-monthly thereafter up until 24 months, and every 6 months thereafter up until 60 months had elapsed. CT of the chest was obtained at 12, 36, and 60 months. Abdominal CT, brain CT and/or magnetic resonance imaging, bone scan, and/or PET scan were performed when disease recurrence was suspected. Details of treatments, their modifications, and clinical evaluation and monitoring schedule of the patients during and after the study therapy are provided in the Online Data Supplement.

After completion/stoppage of the protocol treatment, patients should be followed with no additional therapy until disease relapse. Post-treatment after disease relapse is not regulated by the protocol, either after completion or stoppage of the protocol treatment. No crossover ( $\mathrm{S}-1$ therapy after UFT or vice versa) is allowed. Drug adherence was checked based on the self-report of each patient. Pill counts were not performed.

\section{End Points}

The primary end point was RFS for all randomized patients, which was calculated from the date of registration to the earliest date of disease recurrence or death from any cause. Differentiation of intrapulmonary metastasis (an RFS event) versus second primary lung cancer (not an RFS event) was made clinically by each attending physician, based on tumor histology, differentiation, and vascular invasion, etc. The primary end point was changed from overall survival (OS) to RFS, which was reported to be a surrogate for OS, ${ }^{23}$ by the protocol amendment in February 2014, as described in "protocol amendment" in the Appendix 1 and in the Online Data Supplement itself.

The major secondary efficacy end point was OS, which was measured from the date of registration to the date of death from any cause. Other secondary end points included pattern of recurrence, proportion of treatment completion, oral drug adherence, and the proportions of occurrence of adverse events, severe adverse events, and secondary malignancy.

\section{Statistical Analysis}

The planned sample size was 960 (expected total number of event of 302), which was determined using an accrual period of 5 years, a follow-up period of 5 years, a one-sided type I error of 0.05 , power of $80 \%, 5$-year RFS of $75 \%$ in UFT arm, and hazard ratio (HR) in the S-1 arm of 0.75 (5-year RFS of $80.6 \%$ in the S-1 arm).

A stratified log-rank test was performed in terms of RFS for all randomized patients using stratification factors selected from those used for randomization (pathologic stage [IA/IB] and histology [adenocarcinoma/ not adenocarcinoma]). RFS and OS were estimated using the KaplanMeier method. HRs and their confidence intervals (CIs) were estimated by the Cox proportional hazards model. As a sensitivity analysis, we analyzed RFS and OS in all eligible patients. More details of statistical analysis are given in Online Data Supplement.

\section{RESULTS \\ Patients}

From November 2008 to December 2013, a total of 963 patients were enrolled and underwent randomization, 482 to Arm A (UFT) and 481 to Arm B (S-1). More than 5000 patients were screened for, but did not participate in, the trial. The reasons for non-enrollment and patterns of care of these "non-participants" were collected in an observational study Comprehensive Support Project for Oncology Research (CSPOR) LC-03 and reported elsewhere. ${ }^{24}$

Four patients in UFT arm were ineligible due to advanced (T4) stage, concomitant malignancy, different histology (salivary type), or incomplete lymph node dissection. Five patients in S-1 arm were ineligible due to advanced stage (one T3 patient and two hilar node-positive patients), concomitant malignancy, or incomplete lymph node 


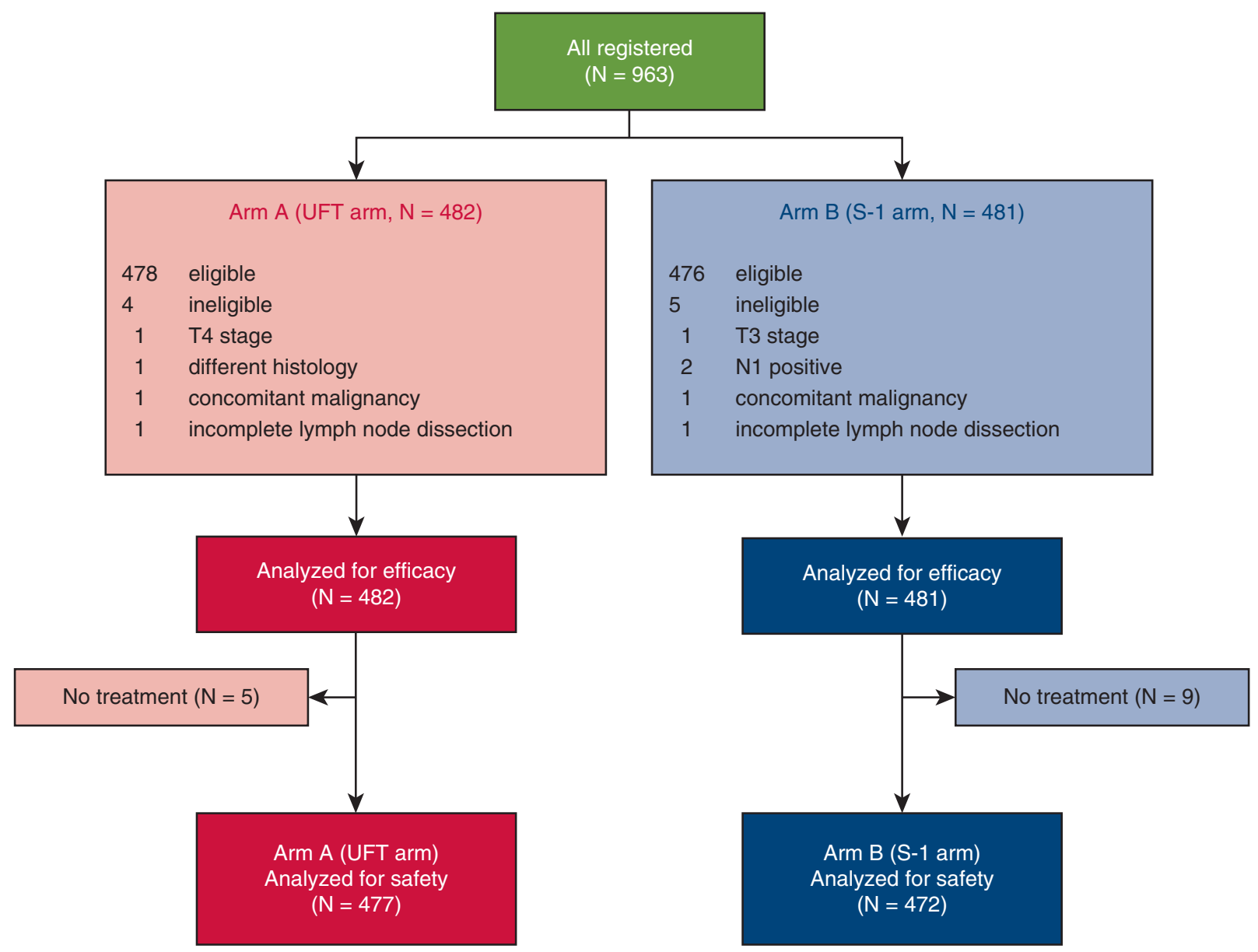

FIGURE 1. CONSORT flowchart of patient selection. Number of enrolled and randomized patients who received treatment and were included in the efficacy and safety analysis. A total of 963 patients were randomized. Four in Arm A and 5 in Arm B were later found ineligible with additional pathologic reports, but they were all included in the efficacy analysis on intent-to-treat basis. Five in Arm A and 9 in Arm B did not receive protocol treatment, and thus were excluded from the safety analysis. UFT, Tegafur/uracil; $S$-1, tegafur/gimeracil/oteracil; N1, hilar node-positive.

dissection. Ineligibilities were revealed with additional pathologic reports after patients were registered and randomized. They were all included in the intention-to-treat efficacy and safety analyses. Five patients in UFT arm and 9 patients in S-1 arm withdrew consent and did not receive the protocol treatment. They were all included in the efficacy analysis, but the safety analysis was performed for a total of 949 patients (477 in UFT arm and 472 in S-1 arm), all of whom received the treatment. Figure 1 shows the patient flow diagram.

Table 1 summarizes the patient characteristics. Information on the molecular change of the tumors, such as epidermal growth factor receptor-activating mutation or anaplastic lymphoma tyrosine kinase gene fusion, was not collected.

\section{Efficacy}

At data cut-off in December 2018, 5 years after the accrual of the last patient, 121 patients in UFT arm and
127 patients in S-1 arm, respectively, died or had disease relapse. Median RFS was not reached in either arm, and the 5-year RFS was $79.4 \%$ (95\% CI, 75.5\%-82.8\%) in UFT arm, and $79.5 \%(95 \% \mathrm{CI}, 75.6 \%-82.9 \%)$ in S-1 arm (Figure 2). The HR of S-1 arm as compared with UFT arm, estimated by a stratified Cox proportional hazards model, was 1.057 (95\% CI, 0.824-1.356) with a stratified, one-sided log-rank test $P$ value of .6684 .

At data cut-off, 77 patients in UFT arm and 85 patients in S-1 arm had died. Median OS was not reached in either arm, and the 5-year OS was $88.8 \%$ (95\% CI, $85.6 \%-91.3 \%$ ) in the UFT arm, and $89.7 \%(95 \%$ CI, $86.6 \%-92.1 \%)$ in the S-1 arm (Figure 3). The HR of the S-1 arm as compared to UFT arm was 1.102 (95\% CI, 0.810-1.500), with onesided $P=.7318$.

There was a significant number of deaths due to causes other than the original lung cancer. In the UFT arm, the original lung cancers accounted for $45(58 \%)$ of the 77 OS events; $30(39 \%)$ died due to other causes, and the cause 
TABLE 1. Patient characteristics

\begin{tabular}{|c|c|c|}
\hline Treatment arm & $\frac{\operatorname{Arm~A~(UFT)~}}{(N=482)}$ & $\frac{\operatorname{Arm} B(S-1)}{(N=481)}$ \\
\hline \multicolumn{3}{|l|}{ Sex } \\
\hline Male & 278 & 279 \\
\hline Female & 204 & 202 \\
\hline Median age, y (range) & $65(37-79)$ & $66(33-80)$ \\
\hline $\begin{array}{l}\text { Median days from surgery } \\
\text { to drug initiation (range) }\end{array}$ & $43(14-66)$ & $42.5(16-67)$ \\
\hline \multicolumn{3}{|l|}{ Histology } \\
\hline Squamous cell carcinoma & 69 & 75 \\
\hline Adenocarcinoma & 386 & 386 \\
\hline Large cell carcinoma & 9 & 9 \\
\hline Other & 18 & 11 \\
\hline \multicolumn{3}{|l|}{ ECOG PS } \\
\hline 0 & 441 & 428 \\
\hline 1 & 41 & 53 \\
\hline \multicolumn{3}{|l|}{ Smoking habit } \\
\hline Never & 193 & 194 \\
\hline Ever & 289 & 287 \\
\hline \multicolumn{3}{|l|}{ Operation procedure } \\
\hline Pneumonectomy & 1 & 1 \\
\hline Lobectomy & 474 & 472 \\
\hline Bi-lobectomy & 7 & 8 \\
\hline \multicolumn{3}{|l|}{ Comorbidities } \\
\hline Present & 295 & 277 \\
\hline Absent & 187 & 204 \\
\hline \multicolumn{3}{|l|}{ Tumor size, cm } \\
\hline$\leq 3$ & 260 & 254 \\
\hline$>3 \sim \leq 4$ & 140 & 149 \\
\hline$>4$ & 82 & 78 \\
\hline \multicolumn{3}{|l|}{ Pathologic $\mathrm{T}$ factor } \\
\hline $\mathrm{T} 1$ & 218 & 226 \\
\hline $\mathrm{T} 2$ & 263 & 254 \\
\hline T3 & 0 & $1 *$ \\
\hline $\mathrm{T} 4$ & $1^{*}$ & 0 \\
\hline \multicolumn{3}{|l|}{ Pathologic $\mathrm{N}$ factor } \\
\hline No & 482 & 479 \\
\hline N1 & 0 & $2 *$ \\
\hline \multicolumn{3}{|l|}{ Pathologic stage } \\
\hline Stage IA & 218 & 224 \\
\hline Stage IB & 263 & 254 \\
\hline Stage IIA & 0 & $2^{*}$ \\
\hline Stage IIB & 0 & $1^{*}$ \\
\hline Stage IIIB & $1^{*}$ & 0 \\
\hline
\end{tabular}

UFT, Tegafur/uracil; $S$-1, tegafur/gimeracil/oteracil; ECOG: Eastern Cooperative Oncology Group; $P S$, Performance Status; NO, node-negative; N1, hilar node-positive. *Ineligible.

of death was unknown in 2 cases. In the S-1 arm, the original lung cancers accounted for $45(53 \%)$ of the 85 OS events; 32 (38\%) died due to other causes, 3 deaths were treatment related, and the cause of death was unknown in 5 cases.
Pre-specified subset analyses for sex, age, smoking, stage, tumor side, lymph node dissection area, pleural invasion, and histology revealed no remarkable results; $\mathrm{S}-1$ was not superior to UFT for either RFS or OS in each analysis (Figures 4 and 5).

Patterns of disease recurrence are summarized as the first relapse sites shown in Table 2. Relapse occurred predominantly as distant metastases, without major imbalances between the arms.

Post-protocol therapy provided for 86 and 91 patients in the UFT and S-1 arms, respectively. Three in the UFT arm and 11 in the $S-1$ arm received post-protocol therapy before documentation of disease recurrence, as deviation from the protocol. In the UFT arm, treatment included chemotherapy in 49 , radiotherapy in 21 , and other modalities in 21 patients; in the S-1 arm, the respective numbers were 54, 31, and 13. Some patients received more than 1 treatment modality.

Figure 6 summarizes the results. RFS and OS were better than expected and not different between the arms, with significant competing risks.

\section{Safety}

In the UFT arm, Grade 3 or greater toxicities were observed in $15.9 \%$ of the patients; $1.5 \%$ experienced hematologic, and $14.7 \%$ non-hematologic toxicities, respectively. In the S-1 arm, Grade 3 or greater toxicities were observed in $14.9 \%$ of the patients; $3.6 \%$ experienced hematologic, and $12.1 \%$ non-hematological toxicities. Details of the toxicities according to the treatment arms are summarized in Table 3. There were 4 patients who died within the protocol, likely due to cardiovascular causes: 1 in the UFT arm and 3 in the S-1 arm. The death of the patient in the UFT arm was due to aortic dissection and adjudicated not to be treatment related, whereas the deaths of patients in the S-1 arm were adjudicated to be treatment-related, probably due to fluorouracil-induced cardiac ischemia. ${ }^{25}$

Toxicities of UFT were unexpectedly much more severe and frequent as compared with previous studies. In the study of Kato and colleagues, ${ }^{7}$ grade 3 or more hepatic toxicities occurred in less than $1 \%$; in the current study, it was $8 \%$. Since they were generally transient, closer follow could have detected asymptomatic abnormalities. However, other toxicities also increased, and exact causes of the discrepancy with previous studies remain unknown.

Dose reductions due to treatment toxicities were performed in $96(20.1 \%)$ patients in the UFT arm, and 190 $(40.3 \%)$ in the S-1 arm, mainly due to gastrointestinal adverse events. Adherence to treatment is summarized in Table 4; 287 patients $(59.5 \%)$ in the UFT arm and 263 $(54.7 \%)$ in the S-1 arm completed the protocol treatment.

During the follow-up period, secondary malignancy developed in $85(17.8 \%)$ in the UFT arm and $84(17.8 \%)$ in the S-1 arm. The most frequent primary site was lung 


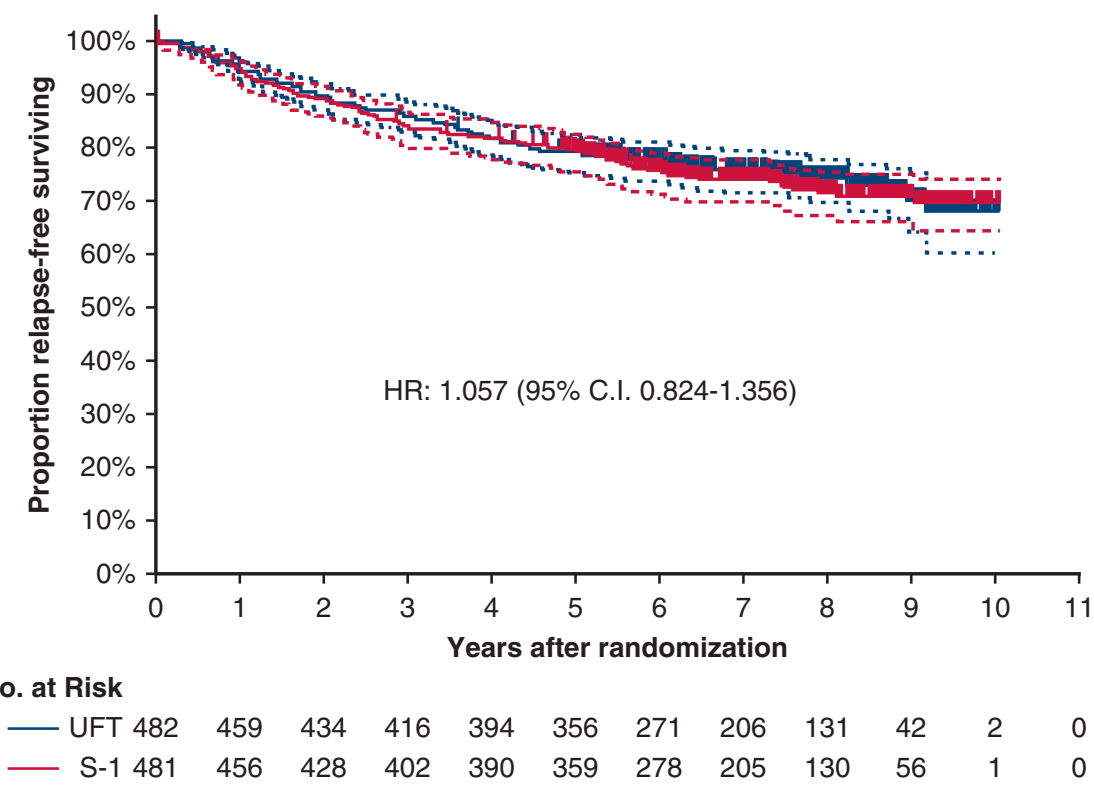

FIGURE 2. RFS of all enrolled and randomized patients on an intention-to-treat basis. Dotted lines express $95 \%$ CIs. There was no significant difference between the arms. The 5-year RFS for the UFT arm (blue lines) was 79.4\%, and that for the S-1 arm (red lines) was 79.5\%. HR, Hazard ratio; CI, confidence interval; UFT, tegafur/uracil; $S$-1, tegafur/gimeracil/oteracil.

in 81 patients, followed by stomach in 23 , prostate in 12 , colorectal in 12, breast in 9 , and bladder in 8 patients.

\section{DISCUSSION}

Optimal management strategy for N0 NSCLC after surgical complete resection remains elusive. Although several guidelines approve the use of platinum-based adjuvant chemotherapy in large $(>4 \mathrm{~cm})$ tumors ${ }^{26,27}$ they are based only on post-hoc subset analyses of randomized trials. ${ }^{2,3}$ While the recent revision of the staging classification of NSCLC upstaged large N0 tumors $(>5 \mathrm{~cm})$ to stage II or $\mathrm{III},{ }^{28}$ there is no direct evidence for the use of platinumbased chemotherapy for the NO subset.

UFT is only marginally active against advanced NSCLC, but, when used in adjuvant settings, was reproducibly shown to improve the postoperative outcome of NO NSCLC

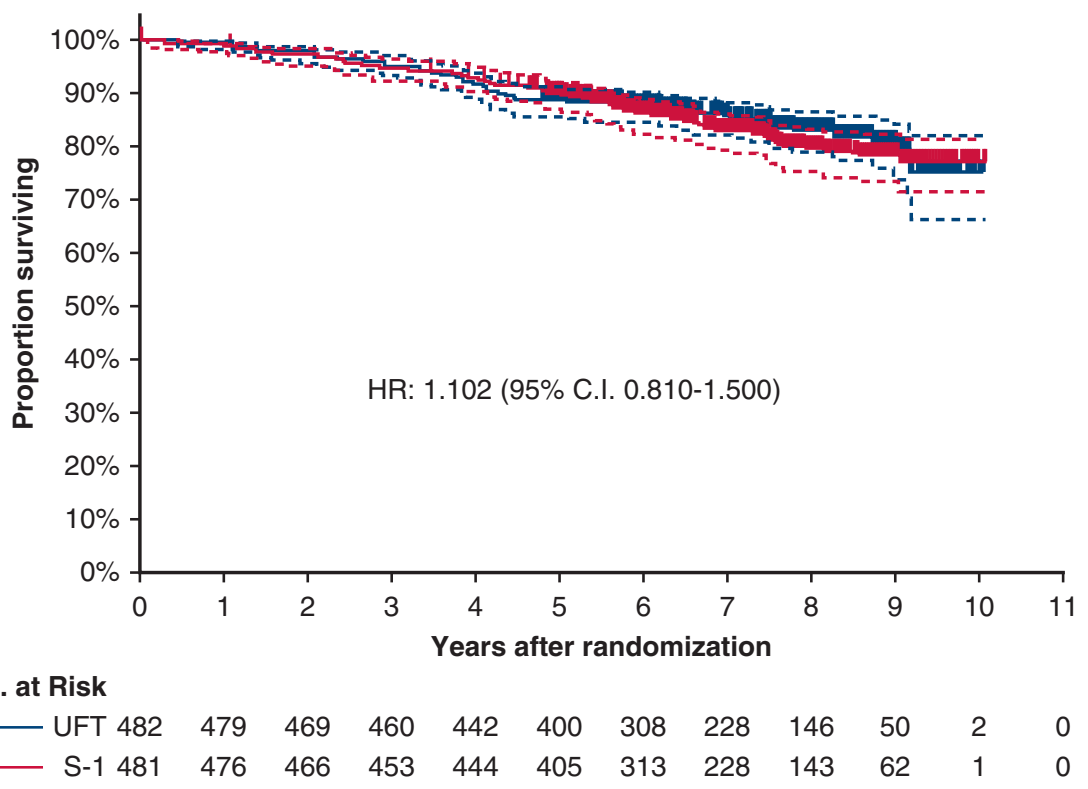

FIGURE 3. OS of all enrolled and randomized patients on an intention-to-treat basis. Dotted lines express $95 \%$ CIs. There was no significant difference between the arms. The 5-year OS for the UFT arm (blue lines) was $88.8 \%$, and that for the S-1 arm (red lines) was $89.7 \%$. HR, Hazard ratio; CI, confidence interval; UFT, tegafur/uracil; $S$-1, tegafur/gimeracil/oteracil. 


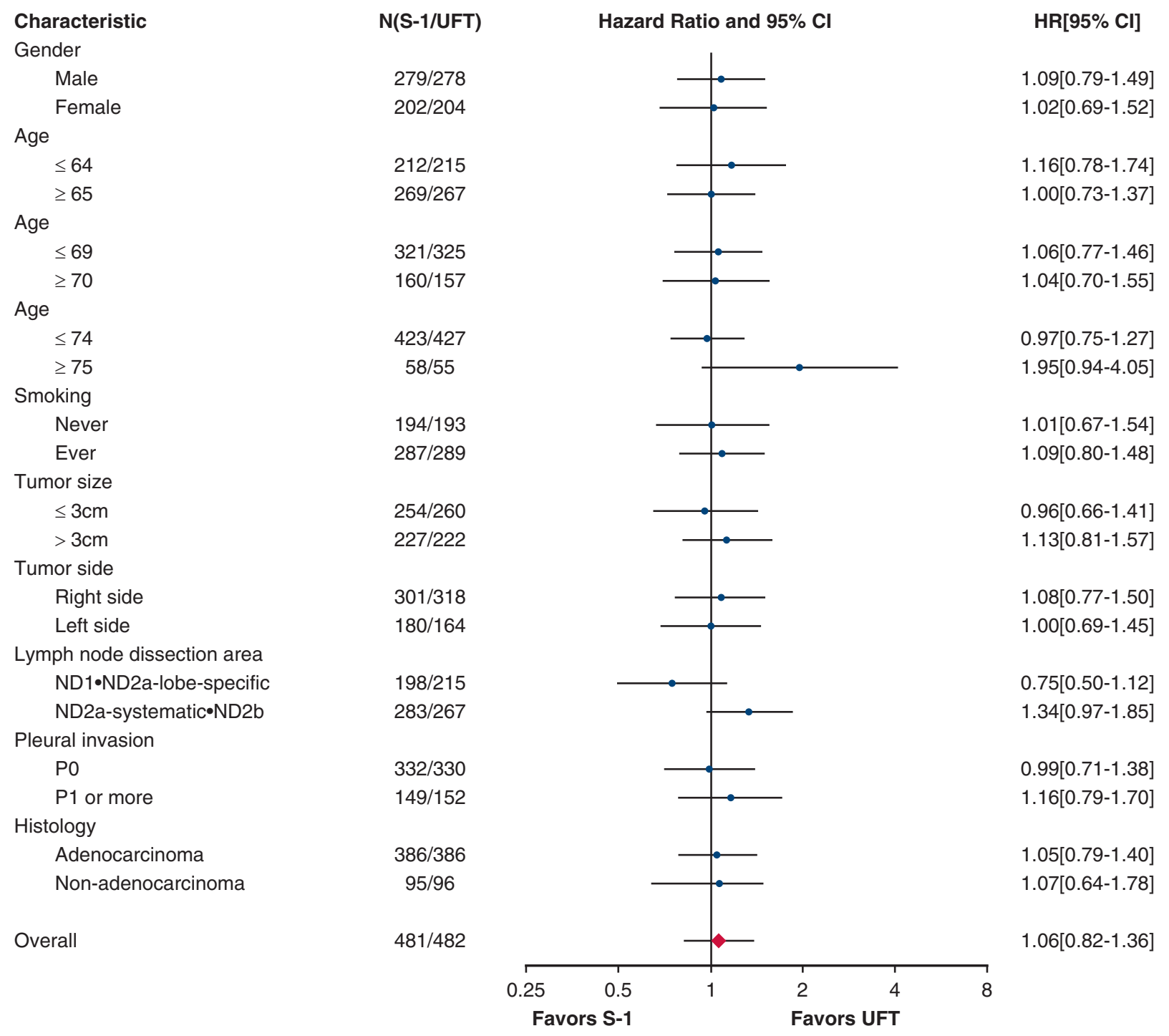

FIGURE 4. Subgroup analysis for relapse-free survival. Forest plot for relapse-free survival according to the potentially prognostic factors in the intentionto-treat population failed to detect any subgroup in which S-1 might be beneficial. $S$-1, Tegafur/gimeracil/oteracil; UFT, tegafur/uracil; $C I$, confidence interval; $H R$, hazard ratio; $N D 1$, hilar lymph node dissection; $N D 2 a$, systemic hilar and mediastinal lymph node dissection excluding anterior and posterior mediastinal nodes; $N D 2 b$, systemic hilar and mediastinal lymph node dissection including anterior and posterior mediastinal nodes.

patients. ${ }^{4-7,10}$ Although its effectiveness was not confirmed in the Western world, it is not that the effect was refuted by Western clinical trials; the trials were never conducted outside Japan. Without any other data against adjuvant UFT effectiveness, we have no other choice but to accept it as a control arm of our trial; a "surgery-only" arm was deemed ethically unacceptable.

$\mathrm{S}-1$ is a newer drug in the same class, combining fluoropyrimidines and DPD inhibition. Its single-agent response rate against advanced NSCLC exceeds $20 \%{ }^{13-15}$ versus less than $10 \%$ in UFT. ${ }^{8,9}$ In fact, postoperative adjuvant therapy with S-1 was reported to be superior to UFT with respect to RFS in rectal cancer. ${ }^{19}$
However, we could not demonstrate the superiority of adjuvant S-1 therapy over UFT in N0 NSCLC. Although the number of events was less than planned even after protocol amendment, there was not even a trend suggesting S-1's superiority. In addition, we could not find any subset, including larger tumors or specific histology, in which S-1 would be beneficial. S-1 was toxic, with 3 treatmentrelated deaths, although none occurred after the protocol amendment to tighten eligibility. The treatment strategy based on risk/benefit in adjuvant therapy for N0 disease could thus be quite different from that with a more advanced tumor; more toxic but active "new-generation drug" might not work well in this population. 


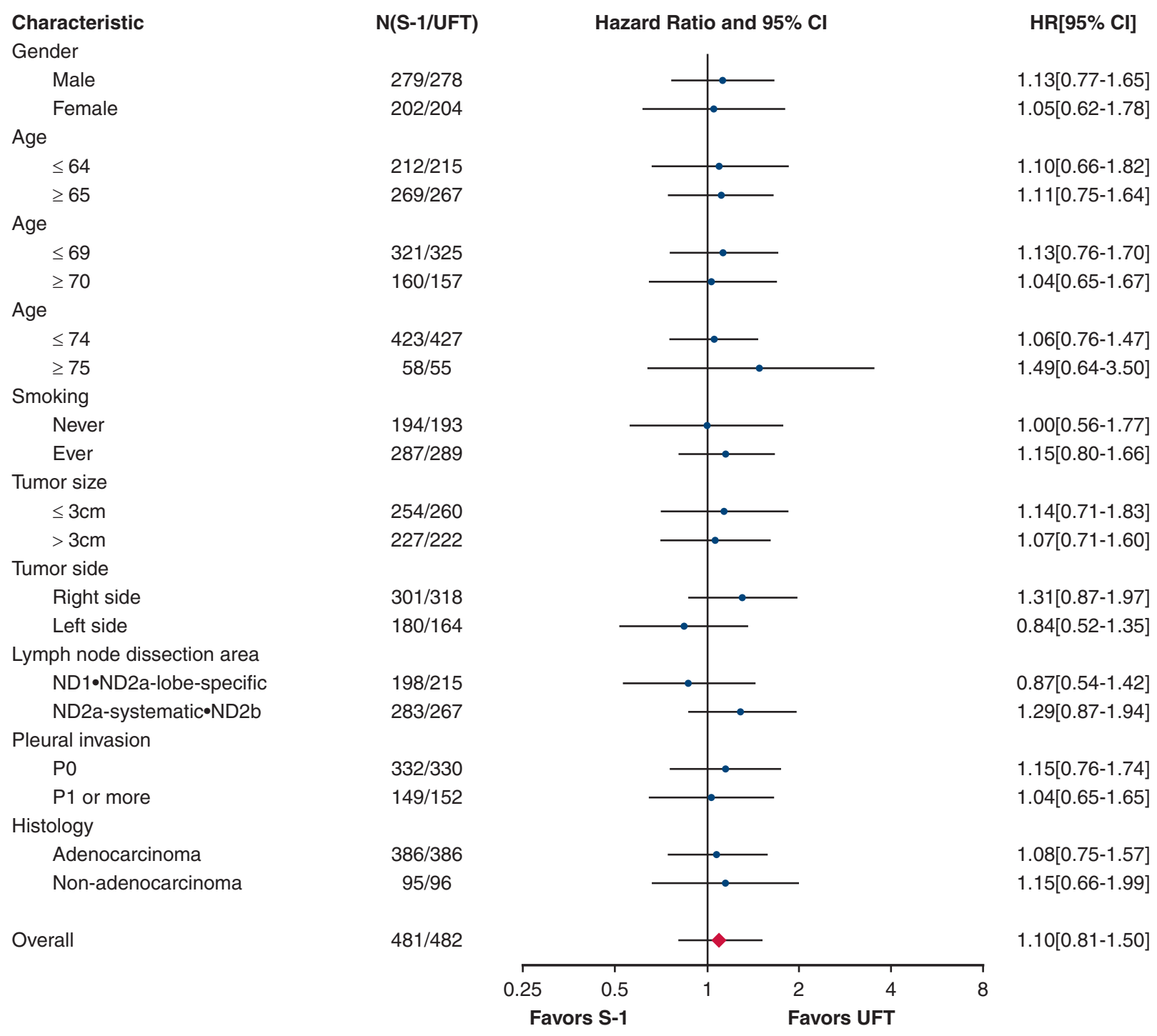

FIGURE 5. Subgroup analysis for overall survival. Forest plot for overall survival according to the potentially prognostic factors in the intention-to-treat population failed to detect any subgroup in which S-1 might be beneficial. S-1, Tegafur/gimeracil/oteracil; UFT, tegafur/uracil; $C I$, confidence interval; HR, hazard ratio; $N D 1$, hilar lymph node dissection; $N D 2 a$, systemic hilar and mediastinal lymph node dissection excluding anterior and posterior mediastinal nodes; $N D 2 b$, systemic hilar and mediastinal lymph node dissection including anterior and posterior mediastinal nodes.

Although our study yielded only negative results, and UFT is not implemented into practice outside Japan, our trial results could nonetheless give some insights and provide suggestions for future management of and research into NO NSCLC.

The outcomes of patients in our trial were exceedingly good for both arms. Although our initial assumption was that the 5-year OS in the UFT arm would be $70 \%$ and that of S-1 would be $76.5 \%$ (HR, 0.75), these turned out to be nearly $90 \%$. This finding is notable because we excluded patients with tumors of $2 \mathrm{~cm}$ or less. We must bear in mind that the prognosis of NO NSCLC, even after excluding "very early" tumors, is excellent, and make future study plans accordingly.
In addition, there was significant competing risk in our study population, with about $40 \%$ of the deceased patients dying from causes other than the original lung cancer. There were thus even fewer disease-specific events. Future trials should therefore incorporate methods to predict who are likely to have disease recurrence. Liquid biopsy might be one method for the identification of these high-risk patients after apparently complete resection. ${ }^{29}$

In contrast, our study revealed that UFT was much more toxic than previously reported, with nearly $15 \%$ of the patients experiencing grade 3 toxicities, most frequently hepatic ones. Although the causes of the discrepancy remain unknown, we might have to re-evaluate the risk/benefit of 
TABLE 2. Initial relapse site

\begin{tabular}{|c|c|c|}
\hline Treatment arm & $\frac{\operatorname{Arm~A~(UFT)~}}{(\mathbf{N}=\mathbf{9 1})^{*}}$ & $\frac{\operatorname{Arm~B~(S-1)~}}{(\mathbf{N}=\mathbf{8 8})^{*}}$ \\
\hline Local & 6 & 5 \\
\hline Hilar or mediastinal lymph node & 23 & 13 \\
\hline Supraclavicular lymph node & 6 & 3 \\
\hline Pleura or pericardium & 15 & 14 \\
\hline Brain & 12 & 18 \\
\hline Pulmonary metastasis & 41 & 37 \\
\hline Other distant metastasis & 32 & 19 \\
\hline
\end{tabular}

UFT, Tegafur/uracil; S-1, tegafur/gimeracil/oteracil. *A patient could have more than 1 site at the disease relapse, and the total number of patients exceeds $100 \%$ of the original population.

our "standard" UFT. With the refinement of the staging system that incorporate invasive tumor size, we should identify patients who could forgo any adjuvant therapy. Observational studies such as CSPOR LC-03 could be useful, and we are now analyzing the UFT effect in the real world. ${ }^{30}$

The fact that $17.8 \%$ of the patients in each arm had secondary malignancies is of some concern, raising the possibility of carcinogenesis from fluoropyrimidine compounds. A previous study by Kato and colleagues ${ }^{7}$ reported that secondary cancers developed much less frequently, in $5 \%$ to $6 \%$ of the cases, and UFT did not appear to influence their development. In contrast, the CSPOR LC-03 observational study found that 797, or $13.5 \%$, of the 5922 patients screened for JCOG0707 had concomitant malignancy and were excluded from the study. ${ }^{24}$ Therefore, it seems that secondary/concomitant malignancies are much more common in our recent patient population.

As in the report of Kato and collegues, ${ }^{7}$ the most frequently observed secondary malignancy was lung cancer. It is often challenging to differentiate a secondary lung cancer from intrapulmonary metastasis. If more intrapulmonary metastases were misdiagnosed as secondary lung cancer than vice versa, the RFS would be overestimated. Future studies may have to incorporate molecular analysis for differentiation of secondary and recurrent tumors.

The strengths of our study are solid study design with randomization, large sample size, and the homogenous patient population of NO NSCLC diagnosed by standard surgical procedures, with the exclusion of early tumors of $2 \mathrm{~cm}$ or less.

The limitations of our study include biologically unexplained (but clinically demonstrated) efficacy of the control UFT arm, better-than-expected patient outcomes leading to fewer events. In retrospect, we underestimated the OS of the control UFT arm, even if we excluded small, early tumors.

A phase III study of adjuvant chemotherapy in patients with completely resected, node-negative NSCLC

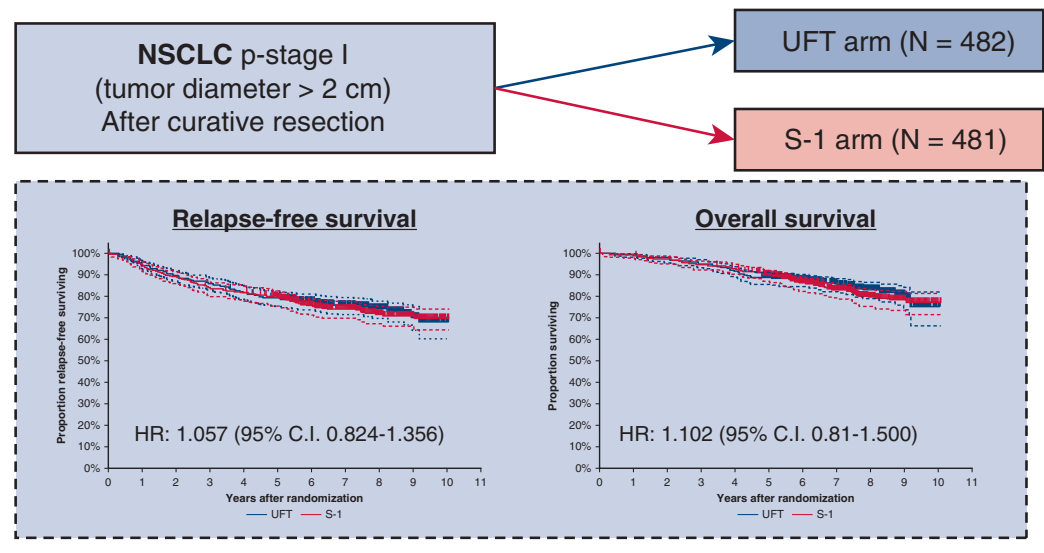

Competing risks

Causes of deaths

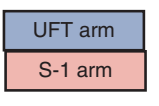

0

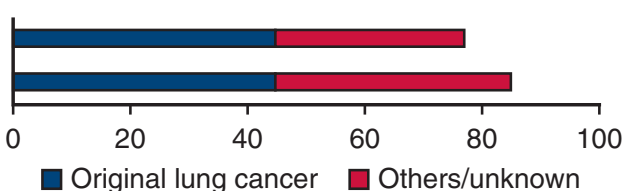

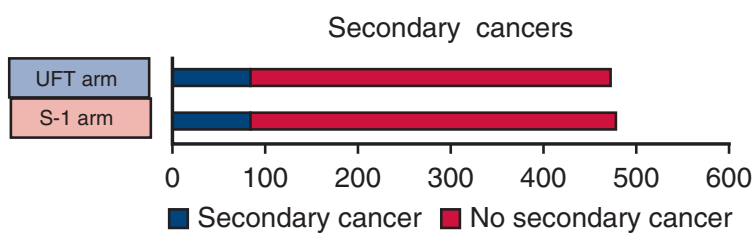

Study design: Phase III randomized trial Primary endpoint: RFS

Results

1) No significant differences in RFS/OS.

2) RFS and OS: far better than expected in both arms.

3) Significant competing risks; further compromising statistical power.

Implications

1) Adjuvant UFT remains standard.

2) Future studies should incorporate identification of high-risk population.

FIGURE 6. In this randomized phase III trial, adjuvant S-1 failed to improve the outcome of patients with node-negative, p-stage I NSCLC as compared with the standard UFT. The RFS/OS were better than expected in both arms, with significant competing risks. Future investigation should incorporate identification of the population at high risk of recurrence. NSCLC, Non-small cell lung cancer; p-stage I, pathological stage I; UFT, tegafur/uracil; S-1, tegafur/ gimeracil/oteracil; RFS, relapse-free survival; $O S$, overall survival, $H R$, hazard ratio; $C I$, confidence interval. 
TABLE 3. Toxicities of the therapy according to study arm

\begin{tabular}{|c|c|c|c|c|c|c|c|c|c|c|c|c|}
\hline & \multicolumn{2}{|c|}{ G1-2 } & \multicolumn{2}{|c|}{ G3 } & \multicolumn{2}{|c|}{ G4 } & \multicolumn{2}{|c|}{ \% Any (G1-4) } & \multicolumn{2}{|c|}{$\%$ G3/4 } & \multicolumn{2}{|c|}{ Missing } \\
\hline & $\frac{\text { Arm A }}{\frac{\text { (UFT) }}{N=477}}$ & $\frac{\frac{\operatorname{Arm~B}}{(\mathrm{S}-1)}}{\mathrm{N}=472}$ & $\frac{\frac{\operatorname{Arm~A}}{(\text { UFT) }}}{\mathrm{N}=477}$ & $\frac{\frac{\text { Arm B }}{(S-1)}}{N=472}$ & $\frac{\frac{\operatorname{Arm~A}}{(\text { UFT) }}}{\mathrm{N}=\mathbf{4 7 7}}$ & $\frac{\frac{\text { Arm B }}{(S-1)}}{\frac{N=472}{N}}$ & $\frac{\overline{\text { Arm A }}}{\frac{\text { (UFT) }}{N=477}}$ & $\frac{\frac{\text { Arm B }}{(S-1)}}{\frac{N=472}{N}}$ & $\frac{\frac{\text { Arm A }}{(\text { UFT) }}}{\frac{N=477}{N}}$ & $\frac{\frac{\text { Arm B }}{(S-1)}}{N=472}$ & $\frac{\overline{\text { Arm A }}}{\frac{\text { (UFT) }}{N=477}}$ & $\frac{\frac{\text { Arm B }}{(S-1)}}{\frac{N=472}{N}}$ \\
\hline \multicolumn{13}{|l|}{$\begin{array}{l}\text { Hematologic } \\
\text { toxicities }\end{array}$} \\
\hline Leukocytes & 47 & 73 & 0 & 2 & 0 & 0 & 9.9 & 15.9 & 0 & 0.4 & & 3 \\
\hline Hemoglobin & 197 & 341 & 1 & 0 & 0 & 1 & 41.5 & 72.5 & 0.2 & 0.2 & & 3 \\
\hline Platelets & 240 & 230 & 0 & 1 & 0 & 0 & 50.3 & 48.9 & 0 & 0.2 & & 3 \\
\hline Neutrophils & 175 & 209 & 6 & 14 & 0 & 1 & 37.9 & 47.5 & 1.3 & 3.2 & 1 & 9 \\
\hline \multicolumn{13}{|l|}{$\begin{array}{l}\text { Non-hematologic } \\
\text { toxicities }\end{array}$} \\
\hline Total bilirubin & 143 & 166 & 0 & 0 & 0 & 0 & 30.0 & 35.2 & 0 & 0 & 1 & 5 \\
\hline AST & 256 & 261 & 24 & 4 & 2 & 0 & 59.1 & 56.1 & 5.5 & 0.9 & & 3 \\
\hline ALT & 205 & 198 & 33 & 7 & 5 & 0 & 50.9 & 43.4 & 8.0 & 1.5 & & 3 \\
\hline Creatinine & 101 & 76 & 0 & 0 & 0 & 0 & 21.2 & 16.1 & 0 & 0 & & 3 \\
\hline Fatigue & 117 & 188 & 2 & 6 & 0 & 0 & 24.9 & 41.1 & 0.4 & 1.3 & & 1 \\
\hline Pigmentation & 51 & 207 & $*$ & $*$ & $*$ & $*$ & 10.7 & 43.9 & $*$ & $*$ & & 1 \\
\hline Rash & 71 & 125 & 1 & 4 & 0 & 0 & 15.1 & 27.3 & 0.2 & 0.8 & & 1 \\
\hline Mucositis, oral & 53 & 138 & 2 & 2 & 0 & 0 & 11.5 & 29.7 & 0.4 & 0.4 & 1 & 2 \\
\hline $\begin{array}{l}\text { Laryngeal } \\
\text { mucositis }\end{array}$ & 9 & 21 & 0 & 0 & 0 & 0 & 1.9 & 4.4 & 0 & 0 & 1 & 2 \\
\hline Anorexia & 128 & 265 & 5 & 15 & 0 & 0 & 27.9 & 59.3 & 1.0 & 3.2 & & 1 \\
\hline Nausea & 83 & 208 & 2 & 5 & 0 & 0 & 17.8 & 45.1 & 0.4 & 1.1 & & 1 \\
\hline Vomiting & 29 & 68 & 0 & 0 & 0 & 0 & 6.1 & 14.4 & 0 & 0 & & 1 \\
\hline Diarrhea & 56 & 145 & 0 & 4 & 0 & 0 & 11.7 & 31.6 & 0 & 0.8 & & 1 \\
\hline
\end{tabular}

$G$, Grade, $U F T$, tegafur/uracil; $S$-1, tegafur/gimeracil/oteracil; $A S T$, aspartate transaminase; $A L T$, alanine transaminase. Asterisk indicates no statistical comparisons were performed between the arms.

Competing risks such as deaths from other causes and secondary malignancies further compromised the statistical power.

\section{TABLE 4. Adherence to the treatment}

\begin{tabular}{|c|c|c|}
\hline Treatment arm & $\frac{\operatorname{Arm~A~(UFT)~}}{(\mathbf{N}=482)}$ & $\begin{array}{c}\text { Arm B (S-1) } \\
(N=\mathbf{4 8 1})\end{array}$ \\
\hline Treatment not started & 5 & 9 \\
\hline Treatment administered & 477 & 472 \\
\hline $\begin{array}{l}\text { Median days of treatment } \\
\text { duration }(25 \%-75 \%)\end{array}$ & $728(281-730)$ & $350(119-369.5)$ \\
\hline $\begin{array}{l}\text { UFT administered for } \\
1 \text { y or more } \\
\% \text { ( } 95 \% \text { confidence } \\
\text { interval })\end{array}$ & $\begin{array}{c}337 / 477 \\
70.7(66.3-74.7)\end{array}$ & \\
\hline $\begin{array}{l}\text { S-1 administered for } \\
0.5 \text { y or more } \\
\% \text { ( } 95 \% \text { confidence } \\
\text { interval })\end{array}$ & & $\begin{array}{c}330 / 472 \\
69.9(65.6-74.0)\end{array}$ \\
\hline $\begin{array}{l}\text { One or more dose reduction } \\
\text { performed }\end{array}$ & $96(20.1 \%)$ & $190(40.3 \%)$ \\
\hline $\begin{array}{l}\text { Protocol treatment } \\
\text { completed } *\end{array}$ & $289(60.0 \%)$ & $263(54.7 \%)$ \\
\hline
\end{tabular}

Other weaknesses of our study design include the lack of masking, lack of uniform staging procedures including use of PET, and no pill counts. Information on detailed tumor size and adenocarcinoma subtype classifications is also lacking, since we used "old" classifications in the 1990s, which were widely used in Japan at the time of the study planning. Also missing are several prognostic factors, including pulmonary function data and detailed comorbidities.

In addition, information of the tumor molecular change, such as epidermal growth factor receptor mutation or anaplastic lymphoma tyrosine kinase infusion, was not collected in this trial. However, surgical resection remains the standard of care for early-stage NSCLC irrespective of its molecular marker, ${ }^{26,27,31}$ and the role of target-based adjuvant therapy is yet to be established, especially in N0 disease. Our results would give benchmark for future research, including trials with target-based drugs or immune-oncology agents.

In conclusion, postoperative adjuvant therapy with oral S-1 was not superior to that with UFT in patients with N0 NSCLC, and UFT remains the standard in this population. Future investigation should incorporate identification of the population at high risk of recurrence (Figure 6). 


\section{Conflict of Interest Statement}

Hideo Kunitoh reports personal fees from Taiho, personal fees from Covidien Japan, personal fees from MSD (Merck Sharp and Dohme), personal fees from EA Pharma, personal fees from Daiichi-Sankyo, personal fees from Johnson and Johnson, personal fees from Boehringer-Ingelheim, personal fees from Eisai, personal fees from AstraZeneca, personal fees from Chugai Pharma, personal fees from Mochida Pharmaceutical, personal fees from Pfizer, personal fees from Takeda, personal fees from Hisamitsu Pharmaceutical, and personal fees from Astellas Pharma, outside the submitted work. Masashiro Tsuboi reports personal fees from AstraZeneca KK, personal fees from Eli Lilly Japan, personal fees from Boehringer-Ingelheim Japan, personal fees from Daiichi-Sankyo, personal fees from Chugai Pharmaceutical CO.,LTD, personal fees from Taiho pharma, personal fees from Johnson \& Johnson Japan, personal fees from Medtronic Japan, personal fees from Teijin Pharma, personal fees from Bristol-Myers Squibb Japan, personal fees from MSD (Merck Sharp and Dohme) Japan, personal fees from Chugai Pharmaceutical CO.,LTD, grants from MSD (Merck Sharp and Dohme) Japan, and grants from Boehringer-Ingelheim Japan, outside the submitted work. Masashi Wakabayashi reports personal fees from Chugai Pharmaceutical Co, Ltd, and personal fees from Johnson and Johnson K. K. Medical Company, outside the submitted work. Morihito Okada reports grants and personal fees from Ono Pharmaceutical company, grants and personal fees from Johnson and Johnson, grants and personal fees from Chugai, grants and personal fees from Covidien Japan, grants from Kyowa Kirin, grants from Daiichi-Sankyo, grants from Taiho, grants from Nihon Medi-Physics, and grants from Nippon Kayaku, outside the submitted work. Kenji Suzuki reports grants and personal fees from Ethicon, grants and personal fees from Medtronic, grants and personal fees from Taiho, grants and personal fees from Eli Lilly, grants and personal fees from Kyowa Kirin, grants and personal fees from Teijin, grants and personal fees from CSL Behring K.K., grants from Shoen kai, grants from Eisai, grants from Shionogi, grants from Ono Pharmaceutical company, personal fees from Intuitive, personal fees from Daiichi-Sankyo, personal fees from Gunze, personal fees from AstraZeneca, and personal fees from ChemoSero-Therapeutic, outside the submitted work. Hisao Asamura reports personal fees from Covidien, personal fees from Johnson and Johnson, grants from Astellas, grants from AstraZeneca, personal fees from Taiho, grants from Meiji, grants from Shionogi, and grants from Ono Pharmaceutical company, outside the submitted work. Shun-ichi Watanabe reported no conflicts of interest.

The Journal policy requires editors and reviewers to disclose conflicts of interest and to decline handling or reviewing manuscripts for which they may have a conflict of interest. The editors and reviewers of this article have no conflicts of interest.

Participating institutions: National Hospital Organization Sendai Medical Center, Tohoku University Hospital, Yamagata Prefectural Central Hospital, Ibaraki Prefectural Central Hospital \& Cancer Center, Tochigi Cancer Center, Gunma Prefectural Cancer Center, Saitama Cancer Center, National Cancer Center Hospital East, Chiba University Graduate School of Medicine, National Cancer Center Hospital, Kyorin University Faculty of Medicine, Tokyo Medical University Hospital, Tokyo Metropolitan Cancer and Infectious Diseases Center Komagome Hospital, Cancer Institute Hospital of Japanese Foundation for Cancer Research, Juntendo University Hospital, Kanagawa Cancer Center, Yokohama Municipal Citizen's Hospital, Yokohama City University Medical Center, Niigata Cancer Center Hospital, Kanazawa University School of Medicine, Saku Central Hospital Advanced Care Center, Shizuoka Cancer Center, Aichi Cancer Center Hospital, Nagoya University School of Medicine, Kyoto University Hospital, Kindai University Hospital, Osaka International Cancer Institute, Osaka Habikino Medical Center, National Hospital Organization KinkiChuo Chest Medical Center, Osaka City General Hospital, Hyogo Cancer Center, Kurashiki Central Hospital, Okayama University Hospital, National Hospital Organization Kure Medical Center and Chugoku Cancer Center, Hiroshima University Hospital, National Hospital Organization Shikoku Cancer Center, National Hospital Organization Kyushu Cancer Center, Fukuoka University School of Medicine, Nagasaki University Hospital, Kumamoto University Hospital, Kumamoto Chuo Hospital, Oita University Faculty of Medicine, National Hospital Organization Okinawa National Hospital.

JCOG Data Center and Operations Office: H. Fukuda (Data Center Director), T. Shibata (Statistics Section), T. Kazato (Data Management Section), T Mizutani and J. Eba, (Operations Office).

\section{References}

1. Pignon JP, Tribodet H, Scagliotti GV, Douillard JY, Shepherd FA, Stephens RJ, et al. Lung adjuvant cisplatin evaluation: a pooled analysis by the LACE collaborative group. J Clin Oncol. 2008;26:3552-9.

2. Strauss GM, Herndon JE II, Maddaus MA, Johnstone DW, Johnson EA, Harpole DH, et al. Adjuvant paclitaxel plus carboplatin compared with observation in stage IB non-small-cell lung cancer: CALGB 9633 with the cancer and leukemia group B, radiation therapy oncology group, and North Central cancer treatment group study groups. J Clin Oncol. 2008;26:5043-51.

3. Butts CA, Ding K, Seymour L, Twumasi-Ankrah P, Graham B, Gandara D, et al. Randomized phase III trial of vinorelbine plus cisplatin compared with observation in completely resected Stage IB and II non-small-cell lung cancer: updated survival analysis of JBR-10. J Clin Oncol. 2010;28:29-34.

4. Wada H, Hitomi S, Teramatsu T. Adjuvant chemotherapy after complete resection in non-small-cell lung cancer. J Clin Oncol. 1996;14:1048-54.

5. Nakagawa M, Tanaka F, Tsubota N, Ohta M, Takao M, Wada H, et al. A randomized phase III trial of adjuvant chemotherapy with UFT for completely resected pathological stage I non-small-cell lung cancer: the west Japan study group for lung cancer surgery (WJSG), the 4th study. Ann Oncol. 2005;16:75-80.

6. Endo C, Saito Y, Iwanami H, Tsushima T, Imai T, Kawamura M, et al. A randomized trial of postoperative UFT therapy in p stage I, II non-small cell lung cancer: North-east Japan study group for lung cancer surgery. Lung Cancer. 2003;40: 181-6.

7. Kato H, Ichinose Y, Ohta M, Hata E, Tsubota N, Tada H, et al. A randomized trial of adjuvant chemotherapy with uracil-tegafur for adenocarcinoma of the lung. $N$ Engl J Med. 2004;350:1713-21. 
8. Keicho N, Saijo N, Shinkai T, Eguchi K, Sasaki Y, Tamura T, et al. Phase II study of UFT in patients with advanced non-small cell lung cancer. Jpn J Clin Oncol. 1986;16:143-6.

9. Ota K, Taguchi T, Kimura K. Report on nationwide pooled data and cohort investigation in UFT phase II study. Cancer Chemother Pharmacol. 1988;22: 333-8.

10. Hamada C, Tanaka F, Ohta M, Fujimura S, Kodama K, Imaizumi M, et al. Metaanalysis of postoperative adjuvant chemotherapy with tegafur-uracil in nonsmall-cell lung cancer. J Clin Oncol. 2005;23:4999-5006.

11. Shirasaka T, Shimamoto Y, Ohshimo H, Yamaguchi M, Kato T, Yonekura K, et al. Development of a novel form of an oral 5-fluorouracil derivative (S-1) directed to the potentiation of the tumor selective cytotoxicity of 5-fluorouracil by two biochemical modulators. Anticancer Drugs. 1996;7:548-57.

12. Shirasaka T, Nakano K, Takechi T, Satake H, Uchida J, Fujioka A, et al. Antitumor activity of $1 \mathrm{M}$ tegafur-0.4 M 5-chloro-2,4-dihydroxypyridine-1 M potassium oxonate (S-1) against human colon carcinoma orthotopically implanted into nude rats. Cancer Res. 1996;56:2602-6.

13. Kawahara M, Furuse K, Segawa Y, Yoshimori K, Matsui K, Kudoh S, et al. Phase II study of S-1, a novel oral fluorouracil, in advanced non-small-cell lung cancer. Br J Cancer. 2001;85:939-43.

14. Nishiyama O, Taniguchi H, Kondoh Y, Takada K, Baba K, Saito H, et al. Phase II study of S-1 monotherapy as a first-line treatment for elderly patients with advanced nonsmall-cell lung cancer: the Central Japan lung study group trial 0404. Anticancer Drugs. 2011;22:811-6.

15. Kasai T, Nakamura Y, Fukuda M, Kitazaki T, Nagashima S, Takatani H, et al. A phase II study of S-1 for previously untreated elderly patients with advanced nonsmall cell lung cancer. Chemotherapy. 2016;61:93-8.

16. Tsukuda M, Kida A, Fujii M, Kono N, Yoshihara T, Hasegawa Y, et al. Randomized scheduling feasibility study of S-1 for adjuvant chemotherapy in advanced head and neck cancer. Br J Cancer. 2005;93:884-9.

17. Sakuramoto S, Sasako M, Yamaguchi T, Kinoshita T, Fujii M, Nashimoto A, et al. Adjuvant chemotherapy for gastric cancer with S-1, an oral fluoropyrimidine. N Engl J Med. 2007;357:1810-20.

18. Uesaka K, Boku N, Fukutomi A, Okamura Y, Konishi M, Matsumoto I, et al. Adjuvant chemotherapy of S-1 versus gemcitabine for resected pancreatic cancer: a phase 3, open-label, randomized, non-inferiority trial (JASPAC 01). Lancet. 2016;388:248-57.

19. Oki E, Murata A, Yoshida K, Maeda K, Ikejiri K, Munemoto Y, et al. A randomized phase III trial comparing S-1 versus UFT as adjuvant chemotherapy for stage II/III rectal cancer (JFMC35-C1: ACTS-RC). Ann Oncol. 2016;27:1266-72.

20. Travis WD, Colby TV, Corrin B, Shimosato Y, Brambilla E. Histological Typing of Lung and Pleural Tumors. 3rd ed. Berlin: Springer-Verlag; 1999.
21. Sobin LH, Wittekind Ch, International Union Against Cancer (UICC). TNM Classification of Malignant Tumors. 5th ed. New York: John Wiley \& Sons, Inc; 1997.

22. Trotti A, Colevas AD, Setser A, Rusch V, Jaques D, Budach V, et al. CTCAE v3.0: development of a comprehensive grading system for the adverse effects of cancer treatment. Semin Radiat Oncol. 2003;13:176-81.

23. Mauguen A, Pignon J-P, Burdett S, Domerg C, Fisher D, Paulus R, et al. Surrogate endpoints for overall survival in chemotherapy and radiotherapy trials in operable and locally advanced lung cancer: a re-analysis of meta-analyses of individual patients' data. Lancet Oncol. 2013;14:619-26.

24. Yoh K, Takamochi K, Shukuya T, Hishida T, Tsuboi M, Sakurai H, et al. Pattern of care in adjuvant therapy for resected Stage I non-small cell lung cancer: realworld data from Japan (Corrigendum: Jpn J Clin Oncol. 2020;50:481). Jpn J Clin Oncol. 2019;49:63-8.

25. Jensen SA, Hasbak P, Mortensen J, Sørensen JB. Fluorouracil induces myocardial ischemia with increases of plasma natriuretic peptide and lactic acid bu without dysfunction of left ventricle. J Clin Oncol. 2010;28:5280-6.

26. NCCN Clinical Practice Guidelines in Oncology (NCCN Guidelines $\left.{ }^{\circledR}\right)$ NonSmall Cell Lung Cancer. Available at: https://www2.tri-kobe.org/nccn/ guideline/lung/english/non_small.pdf. Accessed June 1, 2020.

27. Vansteenkiste J, Crino L, Dooms C, Douillard JY, Faivre-Finn C, Lim E, et al 2nd ESMO consensus conference on lung cancer: early-stage non-small-cel lung cancer consensus on diagnosis, treatment and follow-up. Ann Oncol. 2014;25:1462-74.

28. Brierley J, Gospodarowicz MK, Wittekind C. TNM Classification of Malignant Tumors. 8th ed. Chichester: Wiley Blackwell; 2017.

29. Dasari A, Grothey A, Kopetz S. Circulating tumor DNA-defined minimal residual disease in solid tumors: opportunities to accelerate the development of adjuvant therapies. J Clin Oncol. 2018;36:3437-40.

30. Sakurai H, Goto Y, Yoh K, Takamochi K, Shukuya T, Hishida T, et al. Multicenter observational study of node-negative non-small cell lung cancer patients who are excluded from a clinical trial. J Thorac Oncol. 2019;14:S608-9 (Suppl; abstr P1.17-04)

31. Kris MG, Gaspar LE, Chaft JE, Kennedy EB, Azzoli CG, Ellis PM, et al. Adjuvant systemic therapy and adjuvant radiation therapy for stage I to IIIA completely resected non-small-cell lung cancers: American Society of Clinical Oncology/Cancer Care Ontario clinical practice guideline update. J Clin Oncol. 2017;35:2960-74.

Key Words: non-small cell lung cancer, node-negative, adjuvant chemotherapy, tegafur/uracil, tegafur/gimeracil/ oteracil 


\section{APPENDIX 1 \\ Protocol Amendment}

The initial primary end point was overall survival (OS), with the planned sample size of 960 in total (expected total number of deaths: 302), which was determined with an accrual period of 3 years and a follow-up period of 5 years, using a one-sided type I error of 0.05 , power of $80 \%, 5$-year OS of $70 \%$ in UFT arm, and a hazard ratio (HR) in the S-1 arm of 0.75 (5-year OS of $76.5 \%$ in S-1 arm).

Monitoring in June 2013, after the completion of patient accrual, showed that the combined OS of the 2 arms was far better than expected, with 4-year OS of $91.6 \%$. The original design was thus judged to be underpowered, and the study protocol was amended on February 28, 2014. The primary endpoint was changed to relapse-free survival (RFS), which was reported to be a surrogate for OS. However, the planned sample size was not changed from a total of 960 (expected total number of event of 302), which was re-calculated and determined using an accrual period of 5 years, a follow-up period of 5 years, a one-sided type I error of 0.05 , power of $80 \%, 5$-year RFS of $75 \%$ in UFT arm and HR in the S-1 arm of 0.75 (5-year RFS of $80.6 \%$ in the S- 1 arm). 